\title{
Platelets and pathogens
}

\author{
Kenneth J. Clemetson
}

Received: 5 November 2009/Accepted: 5 November 2009/Published online: 21 November 2009

(c) Birkhäuser Verlag, Basel/Switzerland 2009

Keywords Platelets - Haemostasis - Immunology · Bacteria · Viruses $\cdot$ Parasites $\cdot$ Allergies

Platelets are well recognised as having a critical role in haemostasis, the prevention of bleeding after injury, as well as in the maintenance of the vascular system. In addition, they have a major role in pathological conditions such as thrombosis including cardiac infarction and stroke. Although the normal blood platelet count lies between 150,000 and 350,000 platelets/ $\mu 1$, only a small part of these $(<10,000$ platelets $/ \mu \mathrm{l})$ are actually necessary to prevent bleeding normally, although severe injuries or major surgery may consume more. This suggests that the large numbers of platelets in the circulation have other, additional functions. In evolution, platelets first appeared with the evolution of marsupials and mammals. In earlier vertebrates, such as reptiles and birds, the nucleated thrombocyte covers platelet functions, whereas even further back the haemocyte includes these functions as well as oxygen transport and immunological defence. Evolution from nucleated cells to platelets has involved separation in functions from leukocytes and erythrocytes, specialising in immunology and oxygen transport, respectively. However, there is increasing evidence that platelets carry other residual functions from their common origin. Detailed analysis of receptors expressed by platelets has revealed a wide range of newly discovered molecules including several that are important in

K. J. Clemetson $(\bowtie)$

Theodor Kocher Institute, University of Berne, Freiestrasse 1, 3012 Berne, Switzerland

e-mail: clemetson@tki.unibe.ch immunology, particularly innate immunity. In addition, both in patients and animal models, there is considerable evidence that thrombocytopenia contributes to the severity of diseases caused by pathogens, also supporting a role for platelets in immune defence against these organisms. Although some authors have argued for an important role for reactive oxygen species in platelet defenses against pathogens, others have provided evidence for a strong anti-pathogen effect due to the major participation of the activated platelet in thrombin generation [1].

In the series of papers forming this multi-author review, the authors examine several aspects of platelet function supporting the body's defence against different categories of pathogens. Semple and Freedman [2] review the evidence for the overall role of platelets in innate immunity. Not only do platelets carry critical receptors for the recognition of viruses, bacteria and parasites, but there is increasing evidence that they do indeed have important roles in defence against these pathogens. In addition, platelets interact with many of the major elements of the immune system, including monocytes, dendritic cells and T-lymphocytes and in their storage granules also contain a wide variety of immunologically active substances such as cytokines, chemokines, and related defensins. In addition, they have themselves chemokine receptors, allowing their activation by chemokines released from other platelets or immune cells [3]. CD154 (CD40L) is an important platelet component released on activation that influences the adaptive immune system and interactions with lymphocytes. Recent studies utilizing CD154 knockout mice extend the role of platelet-derived CD154 to the modulation of adaptive immune response by enhancing antigen presentation, improving $\mathrm{CD} 8+\mathrm{T}$ cell responses, and playing a critical function in T-dependent humoral immunity under physiological conditions [4]. 


\section{Platelets and bacteria}

Bacteria are among the many pathogens that interact with platelets, both influenced by platelets and also affecting platelets. Kerrigan and Cox [5] review platelet-bacterial interactions and their role in various diseases, particularly those of the cardiovascular system. Several studies have shown that proteins secreted by or expressed on the surface of specific bacteria can bind to platelets and in some cases activate them. A wide range of different activities have been shown, and it seems likely that interactions such as these have a critical role in bacterial growth and aggression within the cardiovascular system, such as in colonisation and destruction of heart valves. It was suggested many years ago that the early decrease in cardiovascular diseases, observed in the USA from the 1950s on, and long before any decrease in smoking, coincided with the onset of advanced dental hygiene and the systematic removal of dental plaque - a major source of bacteria - by trained hygienists. This link between dental hygiene and circulatory infections has been strengthened by further research, but gum disease remains seriously underestimated as a preventable cause of cardiovascular disease. In some cases bacteria seem able to subvert platelet responses for their own ends. Platelets activated by bacteria can in turn activate endothelial cells and thus exacerbate sepsis.

Yeaman describes the role of platelets in defence against bacteria and the mechanisms they use to achieve this [6]. As well as using innate immunity mechanisms, platelets secrete a large number of granule components that affect bacterial viability. A classic example is the chemokine family member PF4, a strongly basic protein that binds tightly to negatively charged bacteria and induces strong immunological responses. The immunological response to PF4/heparin complexes seen in heparin-induced thrombocytopenia may be an unfortunate consequence of this antibacteria mechanism. Additional platelet anti-bacterial peptides are derived from other chemokine family members by proteolysis and are mainly highly negatively charged molecules. While a number of molecules released from activated platelets have toxic effects on bacteria, activated platelets themselves also affect bacteria by a contact mechanism that may involve internalisation. Among the molecules released from activated platelets are defensins and other microbiocidal peptides. These include the kinocidins, members of the chemokine family, such as platelet factor 4 (PF4, CXCL4) and related structures, that have bacteriocidal characteristics. Other molecules synthesised by activated platelets are hydrogen peroxide and reactive oxygen species, also toxic for bacteria. However, some authors consider that these are minor mechanisms and that the role of platelets in formation of thrombin has a far greater effect on bacteria by generating a strong bactericidal complex in proximity to activated platelet membranes [7].

Platelet-neutrophil extracellular traps are another way in which platelets contribute to anti-bacterial activity by assisting neutrophils to lyse distributing DNA in a "spider's web" that entangles and sticks to bacteria [8].

\section{Platelets and viruses}

Viruses form another important pathogen class. Thrombocytopenia commonly accompanies virus infections that indeed may lead to idiopathic thrombasthenia purpura (ITP) by mechanisms that, as the name suggests, remain obscure. It has proved difficult to demonstrate a direct connection, but research in this area is making progress. Flaujac et al. [9] review the relationship between platelets and viruses, dealing particularly with HIV where interactions with platelets can have serious consequences for public health. Increasing evidence points to platelets playing an important role in anti-viral defence by poorly understood mechanisms. As noted above, platelets are equipped with many of the receptors and immunologically active systems that are critical in immune cells proper. That these also have important functions in platelets is therefore hardly surprising. It should also be noted that severe thrombocytopenia affects many health characteristics other than simply haemostasis.

Recently, there has been increased interest in the role of platelets in dengue, a severe tropical and subtropical viral disease transmitted via a mosquito vector, and with climate warming also more and more a threat to temperate regions. Several papers have provided evidence for dengue virus interactions with platelets, implying that dengue might also be transmittable by platelet transfusions and leaving open the question whether platelets can and do kill dengue virus as part of the host defence [10].

\section{Platelets and parasites}

Throughout most of our evolution humans have been exposed to high parasite loads, and these diseases still remain major causes of mortality in the developing world. Defences against parasites are still poorly understood, although considerable advances have been made over the last few decades. The major parasitic disease affecting humans is malaria, and, despite advances in treatment, between 1 and 3 million people die each year from it, many of them children. One of the most virulent forms is cerebral malaria, which has a poor prognosis. Thus, any advances in understanding its basic pathology may be useful in devising better treatment methods. As part of this multi-author 
review, Cox and McConkey describe the role of platelets in the pathogenesis of cerebral malaria [11].

In a recent important study, purified human platelets have been shown to kill Plasmodium falciparum parasites cultured in red blood cells [12]. Platelet inhibition by aspirin and other platelet inhibitors blocked the lethal effect human platelets exert on $P$. falciparum parasites. These results indicate a protective function for platelets in the early stages of erythrocytic infection distinct from their role in cerebral malaria where they assist infected red blood cells to bind within the cerebral vasculature.

However, platelets may well play a role in defense against other parasites, although this has been poorly studied. Some examples are briefly mentioned. In Lyme disease caused by Borrelia burgdorferi, the platelet count drops, implying a defensive role. The major platelet integrin $\alpha \operatorname{IIb} \beta 3$ is the main receptor for the spirochete [13].

Platelets have also been shown to be capable of killing trypanosomes, though less efficiently than macrophages or neutrophils [14].

Evidence has been obtained for a role for platelets in innate defence against Schistosoma mansoni infections in mice. In replicate experiments, worm burdens were significantly increased in mice thrombocytopenic at the time of infection compared with untreated mice. Induction of thrombocytopenia 4 days after infection had no effect on worm count. Platelets from non-immune mice adhere to and kill mechanically transformed schistosomula in vitro. Platelets may thus provide an innate defence against schistosome infection, and the thrombocytopenia that occurs during schistosome infections may be a strategy that helps secondary parasites evade this type of host defence or could reflect platelet losses during the defence reaction [15].

There is also evidence that platelets are involved in patients infected with Echinococcus granulosus. The concentrations of $\beta$-thromboglobulin and platelet factor 4 in infected patients were elevated compared with controls, indicating that platelets had been activated [16].

In many of these parasitic diseases, it has been suggested that the platelet $\mathrm{IgE}$ receptor has an important role. Flow cytometry analysis showed that, like monocytes and eosinophils, about $10-20 \%$ of platelets in normal individuals are IgE-positive and that this increases to up to $50 \%$ in patients with parasitic diseases, leading to increased levels of circulating IgE, particularly in filariasis [17]. Binding of IgE was reduced by anti-IgE receptor antibodies, and Fc\&RI was later shown to be present on platelets with about 8001,000 Fc «RI molecules per platelet [17] (1). Parasite-specific IgE binding to platelets followed by platelet activation when these are cross-linked by binding to the parasites is thought to be the major mechanism involved. As a result, platelets produce oxygen metabolites and release granule contents toxic for the parasite.
Platelets, parasites and allergies

Before the industrial era, all mammals were 'normally' infected with helminth parasites from shortly after birth until adulthood. Only since the nineteenth century have people living in developed countries organised sophisticated water purification (chlorination) and sewage disposal systems that have allowed them to escape helminth infection. Allergies are present at high levels in these human populations as they are absent in populations still regularly exposed to helminths. In addition, populations with endemic helminth infections show little allergic pathology that could be connected to the acquisition or elimination of helminth parasites. It has therefore been suggested that endemic helminth infections activate the Th2 system, particularly at mucosal surfaces, to provide a different level of immunological homeostasis than occurs at present in developed societies. Under these conditions, mast cells, eosinophils and IgE rarely participate in allergic reactions, although they obviously participate in the control of helminth infections. Allergic reactions can thus be considered to be a pathologic consequence of the change in this homeostatic mechanism and have no protective consequences for the individual [18]. The realization that this reciprocal relationship exists may open new approaches to prevention of allergic disorders, which are becoming an increasingly major problem in developed countries.

Some recent studies suggest that platelets may play an important role in allergic inflammation through the high affinity $\operatorname{IgE}$ receptor $(\mathrm{Fc} \varepsilon \mathrm{RI})$, the low affinity $\operatorname{IgE}$ receptor (Fc\&RII/CD23) and the low affinity IgG receptor (Fc $\gamma$ RIIA/ CD32) expressed on the cell surface. Human platelets released serotonin and the chemokine RANTES (regulated upon activation, normal $\mathrm{T}$ expressed and presumably secreted), induced via Fc\&RI, but the biological implication of human platelets in type I allergy is not yet clear. The levels of RANTES released from platelets from allergic patients and healthy individuals stimulated with monoclonal antibody $(\mathrm{Ab})$ to human $\mathrm{F} \varepsilon \varepsilon \mathrm{RI} \alpha$-chain or human myeloma IgE and anti-human IgE Ab were compared. The level of RANTES released from platelets of allergic patients stimulated with human IgE and anti-human $\operatorname{IgE}$ was significantly higher than that of healthy individuals. While the surface expression levels of Fc\&RI on the platelets from allergic patients and healthy individuals were not significantly different, the platelets from allergic patients were more activated by the IgE-Fc $\&$ RI pathway than those from healthy controls, suggesting a novel and important role for human platelets in perpetuating allergic inflammation through IgE and the FceRI [19].

Other studies indicated that both the presence of functional, intact platelets and the release of inflammatory mediators from platelets are crucial events for a sustained 
allergic inflammatory response. Recently, platelets were shown to be critical for leukocyte recruitment in chronic skin inflammation in a mouse model of chronic contact dermatitis. Using platelet depletion and restitution in an IgE-mediated intermediate hypersensitivity reaction in skin, the effect of mediators released from activated platelets on leukocyte recruitment was investigated [20].

\section{References}

1. Zander DM, Klinger M (2009) The blood platelets contribution to innate host defense-what they have learned from their big brothers. Biotechnol J 4:914-926

2. Semple JW, Freedman J (2010) Platelets and innate immunity. Cell Mol Life Sci (this issue)

3. Clemetson KJ, Clemetson JM, Proudfoot AE, Power CA, Baggiolini M, Wells TN (2000) Functional expression of CCR1, CCR3, CCR4, and CXCR4 chemokine receptors on human platelets. Blood 96:4046-4054

4. Sprague DL, Sowa JM, Elzey BD, Ratliff TL (2007) The role of platelet CD154 in the modulation in adaptive immunity. Immunol Res 39:185-193

5. Kerrigan SW, Cox D (2010) Platelet-bacterial interactions. Cell Mol Life Sci (this issue)

6. Yeaman MR (2010) Platelets in defense against bacterial pathogens. Cell Mol Life Sci (this issue)

7. Sun H, Wang X, Degen JL, Ginsburg D (2009) Reduced thrombin generation increases host susceptibility to group A streptococcal infection. Blood 113:1358-1364

8. Clark SR, Ma AC, Tavener SA, McDonald B, Goodarzi Z, Kelly MM, Patel KD, Chakrabarti S, McAvoy E, Sinclair GD, Keys EM, Allen-Vercoe E, Devinney R, Doig CJ, Green FH, Kubes P (2007) Platelet TLR4 activates neutrophil extracellular traps to ensnare bacteria in septic blood. Nat Med 13:463-469

9. Flaujac C, Boukour S, Cramer Bordé E (2010) Platelets and viruses: an ambivalent relationship. Cell Mol Life Sci (this issue)
10. Ghosh K, Gangodkar S, Jain P, Shetty S, Ramjee S, Poddar P, Basu A (2008) Imaging the interaction between dengue 2 virus and human blood platelets using atomic force and electron microscopy. J Electron Microsc (Tokyo) 57:113-118

11. Cox D, McConkey S (2010) The role of platelets in the pathogenesis of cerebral malaria. Cell Mol Life Sci (this issue)

12. McMorran BJ, Marshall VM, de Graaf C, Drysdale KE, Shabbar M, Smyth GK, Corbin JE, Alexander WS, Foote SJ (2009) Platelets kill intraerythrocytic malarial parasites and mediate survival to infection. Science 323:797-800

13. Alugupalli KR, Michelson AD, Barnard MR, Robbins D, Coburn J, Baker EK, Ginsberg MH, Schwan TG, Leong JM (2001) Platelet activation by a relapsing fever spirochaete results in enhanced bacterium-platelet interaction via integrin $\alpha \operatorname{Ilb} \beta 3$ activation. Mol Microbiol 39:330-340

14. Shaw KT, Mawji Y, Stevenson MM, Kongshavn PA (1991) Cells within the vascular system capable of mediating trypanocidal activity in vitro. Infect Immun 59:3143-3150

15. Stanley RG, Ngaiza JR, Wambayi E, Lewis J, Doenhoff MJ (2003) Platelets as an innate defence mechanism against Schistosoma mansoni infections in mice. Parasite Immunol 25:467473

16. Matowicka-Karna J, Kemona H, Dymicka-Piekarska V, Butkiewicz A (2005) The secretory activity of blood platelets- $\beta$ thromboglobulin and platelet factor 4 in echinococcosis. Pol Merkur Lekarski 19:172-174

17. Joseph M, Capron A, Ameisen JC, Capron M, Vorng H, Pancre V, Kusnierz JP, Auriault C (1986) The receptor for IgE on blood platelets. Eur J Immunol 16:306-312

18. Bell RG (1996) IgE, allergies and helminth parasites: a new perspective on an old conundrum. Immunol Cell Biol 74:337-345

19. Hasegawa S, Tashiro N, Matsubara T, Furukawa S, Ra C (2001) A comparison of FceRI-mediated RANTES release from human platelets between allergic patients and healthy individuals. Int Arch Allergy Immunol 125(suppl 1):42-47

20. Tamagawa-Mineoka R, Katoh N, Kishimoto S (2009) Platelets play important roles in the late phase of the immediate hypersensitivity reaction. J Allergy Clin Immunol 123:581-587 